\title{
Durabilité des composites pour énergie marine renouvelable
}

\author{
P. Davies ${ }^{1}$, A. Boisseau1', D. Choqueuse ${ }^{1}$, F. Thiebaud ${ }^{2,3}$ et D. Perreux ${ }^{2,3}$ \\ 1 Ifremer, Service Matériaux et Structures, 29280 Plouzané, France \\ e-mail : peter.davies@ifremer.fr \\ 2 MaHyTec, 210, avenue de Verdun, 39100 Dole, France \\ 3 Université de Franche-Comté, LMARC/FEMTO-ST, 25000 Besançon, France
}

\section{Mots-clés :}

Fatigue ; eau de mer ; énergie marine; hydrolienne; pale

Key words:

Fatigue; sea water; ocean energy; tidal turbine; blade

\begin{abstract}
Résumé - Les matériaux composites joueront un rôle essentiel dans le développement de systèmes de conversion d'énergie marine renouvelable, et pour cette application compte tenu des contraintes liées à la maintenance une excellente durabilité à long terme s'avère indispensable. On dispose d'une bonne expérience de ces matériaux en milieu marin, et pour des applications, telles les éoliennes, où ils sont soumis à un chargement cyclique, mais on ne trouve que très peu d'informations sur leur comportement sous sollicitation cyclique dans l'eau de mer. Cet article adresse cette lacune, et présente les résultats d'une étude du comportement sous chargement quasi-statique et cyclique de composites renforcés de différentes fibres de verre et sollicités dans l'eau de mer. Pour ces conditions de chargement couplé, le type de fibre, la résine et l'interface fibre/matrice influent tous sur la durée de vie.
\end{abstract}

\begin{abstract}
Durability of composites for renewable ocean energy. Composites will play an essential role in the development of ocean energy conversion systems, and their long term durability is critical to the success of these structures. Composites have been widely used in marine applications and in structures highly loaded in fatigue such as wind turbine blades. However, very few data are available to evaluate their performance under conditions which combine long term immersion in sea water and sustained cyclic loading. In this study composites reinforced with three different types of glass fibre and the same epoxy resin have been tested under these conditions, and the influence of aging on both static and fatigue behaviour has been quantified. Fibre type, resin and fibre/resin interface all influence the fatigue behaviour of these materials in sea water.
\end{abstract}

Reçu le 27 septembre 2011 accepté le 11 janvier 2012 liennes) le potentiel techniquement exploitable mondial est estimé à $450 \mathrm{TWh} / \mathrm{an}$, (il faut noter néanmoins que les estimations à l'échelle mondiale varient de 100 TWh par an à plus de $3.500 \mathrm{TWh}$ !). Celui de l'Europe serait compris entre 15 et $35 \mathrm{TWh} / \mathrm{an}$, pour 10 GW installés. Le potentiel techniquement exploitable français serait compris entre 5 et $14 \mathrm{TWh} /$ an selon EDF, pour 2,5 à 3,5 GW installés. Les sites potentiels sont spécifiques (détroits, caps, goulets où l'on observe une augmentation des vitesses) et bien identifiés (en France : Raz Blanchard, Fromveur, Raz de Sein, Héaux de Bréhat, Raz de Barfleur. . . et en Outre Mer).

L'exploitation de ces énergies est à différents stades de développement mais les conditions d'exploitation en mer rendront 
difficile l'accès pour les opérations de maintenance ou réparation. Les matériaux composites devraient jouer un rôle important dans ce développement, grâce à leurs propriétés spécifiques élevées et une bonne résistance au vieillissement [4]. Pour cela, il faut impérativement s'assurer de leur durabilité, la rentabilité d'un système de conversion d'énergie marine nécessitant une fiabilité accrue. Compte tenu du coût élevé des interventions en mer, un fonctionnement sans intervention lourde est à garantir sur une durée d'au moins 5 ans. L'IFREMER s'intéresse aux énergies marines renouvelables (EMR) depuis de nombreuses années, travaillant sur l'énergie thermique des océans depuis plus de 20 ans, mais depuis quelques années ce sujet est devenu un des axes prioritaires de l'Institut. Ainsi, des thèmes de recherche aussi divers que l'impact environnemental et l'évaluation hydrodynamique de prototypes sont regroupés dans un programme de recherche, et un document de référence a été rédigé [1]. L'étude des matériaux, et en particulier la durabilité des matériaux composites pour ces applications, fait partie de cet effort.

\section{Les composites dans l'eau de mer}

On utilise les matériaux composites pour les bateaux de plaisance depuis plus de 50 ans [5,6], et l'influence de l'eau sur les propriétés des composites a fait l'objet de nombreuses études [7-9]. Les études portant sur le comportement en eau de mer sont plus rares, quelque unes sont toutefois relevées [par ex. 10-13] et conduisent à constater que selon le matériau et les conditions d'essai, l'eau de mer peut entraîner une diminution importante des propriétés mécaniques.

\section{La fatigue des composites}

Une littérature abondante sur la fatigue des composites est disponible, Harris a présenté un état de l'art en 2003 [14]. On trouve des résultats pour une grande diversité de matériaux, de géométries des éprouvettes, et de conditions d'essai. Le développement des éoliennes a permis de financer des études importantes, notamment aux USA $[15,16]$ et en Europe $[17,18]$ sur cette thématique. Pour le projet OPTIMAT, par exemple, plus de 3000 éprouvettes ont été testées, alimentant une base de données importante [19]. On constate néanmoins qu'il n'y a que peu d'essais normalisés dans ce domaine.

\section{L'utilisation des composites pour l'énergie marine renouvelable}

Bien que l'on soit aux débuts de la génération d'électricité à partir des vagues et des courants, il existe quelques prototypes en mer. Dans le projet Seaflow, de Marine Current Turbines (MCT), le leader dans ce domaine, une hydrolienne de $300 \mathrm{~kW}$ avec 2 pales en composite a été immergée près de la côte du Devon en Angleterre en juin 2003. Les pales étaient fabriquées à partir d'un noyau central de $65 \mathrm{~mm}$ d'épaisseur en carbone/époxy recouvert d'une enveloppe en verre/époxy [20]. En 2003, une hydrolienne de $300 \mathrm{~kW}$ à 3 pales en composite a été installée en Norvège à Hammerfest. En 2008, dans le projet SeaGen, une doubleturbine de 1,2 MW a été immergé en Irlande, avec une conception similaire à celle de Seaflow mais avec des pales plus longues de 7,5 mètres [21]. En février 2010, plus de 350 MWh d'électricité avaient été fournis au réseau. En avril 2011, MCT a annoncé l'installation de 4 hydroliennes au nord de l'Ecosse en 2014 d'une puissance de $8 \mathrm{MW}$.

Aux États Unis dans le projet RITE, six turbines à pales composites ont été installées en 2007 dans l'East River à New York. Suite à quelques ruptures de pales la conception a dû être modifiée. Des pales en composite sont également employées sur l'hydrolienne d'OpenHydro mais de récentes informations font état de la rupture de toutes les pales, l'année dernière lors des essais dans une zone à fort courant au Canada [22]. Ceci souligne la sévérité de l'environnement et la nécessité de bien dimensionner les pales d'hydroliennes.

En France la société HydroHelix a mis à l'eau, en 2008, un prototype Sabella à 6 pales le D03 (diamètre 3 mètres), figure 1a [23].

Un nouveau projet, financé par l'ADEME, pour une turbine de diamètre 


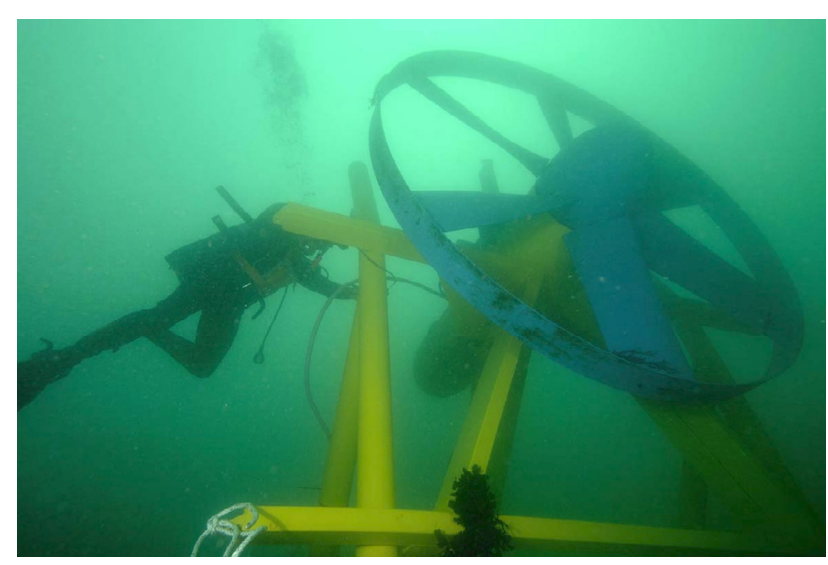

(a)

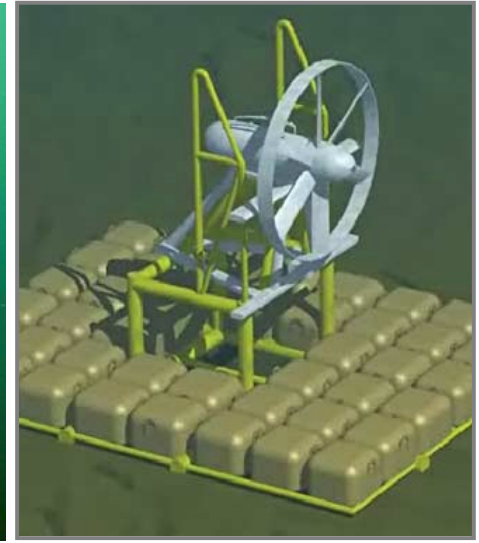

(b)

Fig. 1. (a) Hydrolienne Sabella D03 en immersion, crédit photo : SABELLA - GLADU - BALAO. (b) Projet Sabella D10.

Fig. 1. (a) Sabella D03 tidal tubine, (c) SABELLA - GLADU - BALAO. (b) Sabella D10 project.

10 mètres (D10) est en cours de réalisation, figure $1 \mathrm{~b}$. L'hydrolienne devrait être installée dans le Fromveur en Bretagne en fin d'année 2011. D'autres développements en France sont en cours, notamment dans le cadre du projet Harvest (Hydrolienne à Axe de Rotation Vertical stabilisé) développé à Grenoble, avec une hydrolienne à axe vertical $[24,25]$.

Pour ce type d'applications, on note également l'utilisation des composites pour la réalisation de carénages et de cadres de support, en raison de leur faible poids (installation et maintenance facilitées). L'utilisation des composites est également envisagée pour les systèmes houlomoteurs [26].

\section{5 Étude de durabilité}

Les paragraphes 3 et 4 soulignent l'existence de bases de données importantes concernant le comportement des composites dans l'eau et leur comportement en fatigue. Par contre, il n'y a que très peu de données pour décrire la réponse de composites soumis à des chargements cycliques dans l'eau de mer $[27,28]$. Dans ce contexte, une étude spécifique a été menée depuis quatre ans sur la durabilité des composites en milieu marin, dont une partie a été présentée par Boisseau [29,30]. Les objectifs de cette étude étaient tout d'abord de caractériser le comportement de composites renforcés de fibres de verre sous chargement cyclique, dans l'eau de mer, et ceci afin d'établir une base de données qui pourrait être utilisée dans la conception de ces structures. Ensuite, en utilisant ces données et celles d'une campagne d'essais de vieillissement accéléré en immersion, un modèle du comportement du matériau a été établi. Celui-ci a été intégré dans un modèle de structure EMR, dans un premier temps une hydrolienne a été modélisée. Des essais de turbine à l'échelle réduite en bassin ont complété l'étude.

\subsection{Matériaux}

Cette étude s'est surtout focalisé sur l'influence du type de fibres de verre sur le comportement des composites. Trois fibres ont été étudiées, tableau 1 . Le verre $E$ est la fibre de référence. Le verre Advantex est une fibre sans bore, formulée pour une meilleure résistance à la corrosion. Le verre HiPer-tex est une fibre en verre $R$ haute performance, également formulée sans bore.

Les renforts fournis par $O C V$ et $3 B$ sont quasi-unidirectionnels, (CDM 1250, avec $1150 \mathrm{~g} / \mathrm{m}^{2}$ de fibres à $0^{\circ}, 50 \mathrm{~g} / \mathrm{m}^{2}$ à $90^{\circ}$ et $50 \mathrm{~g} / \mathrm{m}^{2}$ de mat). La résine employée est l'époxy MGS ${ }^{\odot}$ RIM 135 de Momentive (ex Hexion) avec un durcisseur amine RIMH 137. Cette résine et ce type de renfort sont actuellement utilisés pour fabriquer des pales d'éoliennes. Quelques essais avec une autre formulation de résine et un ensimage non-optimisé ont également été réalisés, 
Tableau 1. Fibres de verre étudiées, propriétés en traction (données fournisseurs, ASTMD2343). Table 1. Glass fibres studied, tensile properties (suppliers' data, ASTM D2343).

\begin{tabular}{lccc}
\hline & Référence & Module [GPa] & Résistance [MPa] \\
\hline Verre $E$ & $E$ & $74-80$ & $2000-2500$ \\
\hline Advantex $^{\circledR}$ & ADV & $81-83$ & $2200-2600$ \\
\hline HiPertex $^{\text {TM }}$ & HP & $89-91$ & $2800-2900$ \\
\hline
\end{tabular}

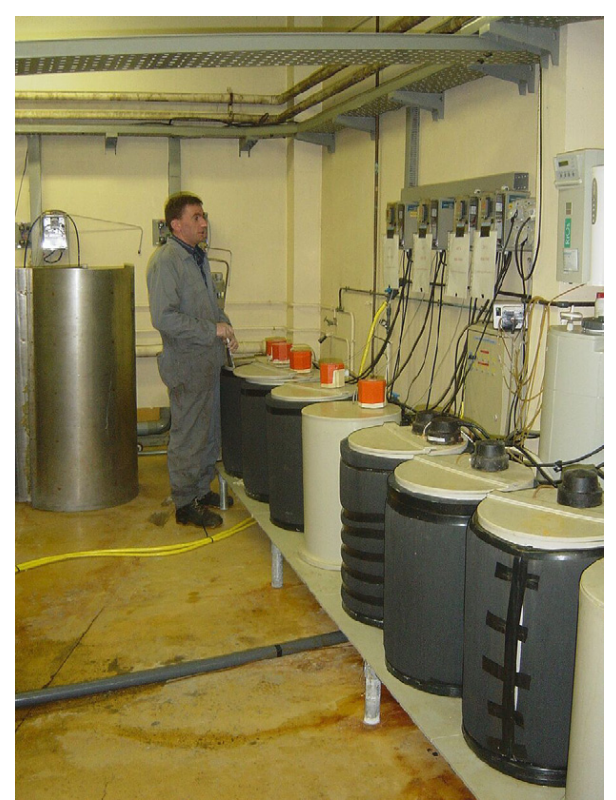

(a)

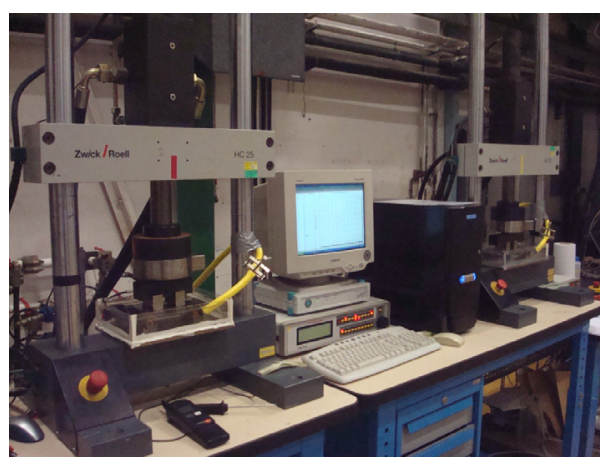

(b)

Fig. 2. Moyens d'essai, (a) vieillissement, et (b) fatigue dans l'eau de mer.

Fig. 2. Test facilities, (a) ageing, (b) fatigue in sea water.

afin d'étudier l'influence de ces paramètres. Toutes les éprouvettes ont été fabriquées par infusion à $35{ }^{\circ} \mathrm{C}$ dans une enceinte climatique et post-cuites pendant $10 \mathrm{~h}$ à $70^{\circ} \mathrm{C}$.

\subsection{Moyens d'essais}

Les moyens d'essais employés pour cette étude sont des bacs d'eau de mer naturelle, renouvelée, contrôlée en température $(4,20$, 40 , $60{ }^{\circ} \mathrm{C}$ ), figure $2 \mathrm{a}$, des machines d'essais classiques et des bancs d'essais de fatigue dans l'eau de mer, (également renouvelée et à température contrôlée) figure $2 b$.

La plupart des essais ont été réalisés sur des éprouvettes en forme haltère, figure 3, en flexion 4 points. Ce choix a été dicté par les résultats d'une étude préliminaire. Une sollicitation en flexion quatre points a été adoptée afin de pouvoir appliquer des chargements en traction, compression et cisaille-

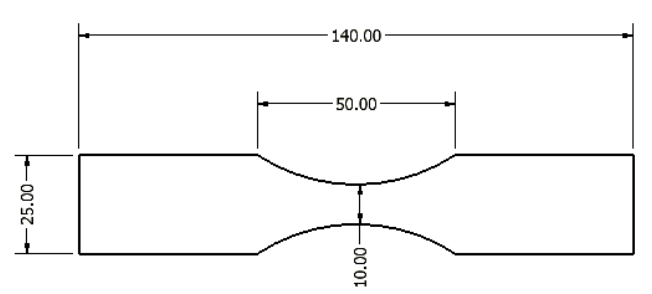

Fig. 3. Géométrie d'éprouvette, dimensions en millimètres.

Fig. 3. Specimen geometry, dimensions in millimeters.

ment en même temps et de pouvoir réaliser ces essais en immersion. L'éprouvette parallèle de forme normalisée a tendance à casser prématurément par poinçonnement sous les points de chargement. En réduisant la section de la partie centrale des ruptures valables sont obtenues à des niveaux de contrainte plus élevés. Les éprouvettes ont été découpées par jet d'eau dans des plaques infusées, ce qui fournit un bon état 

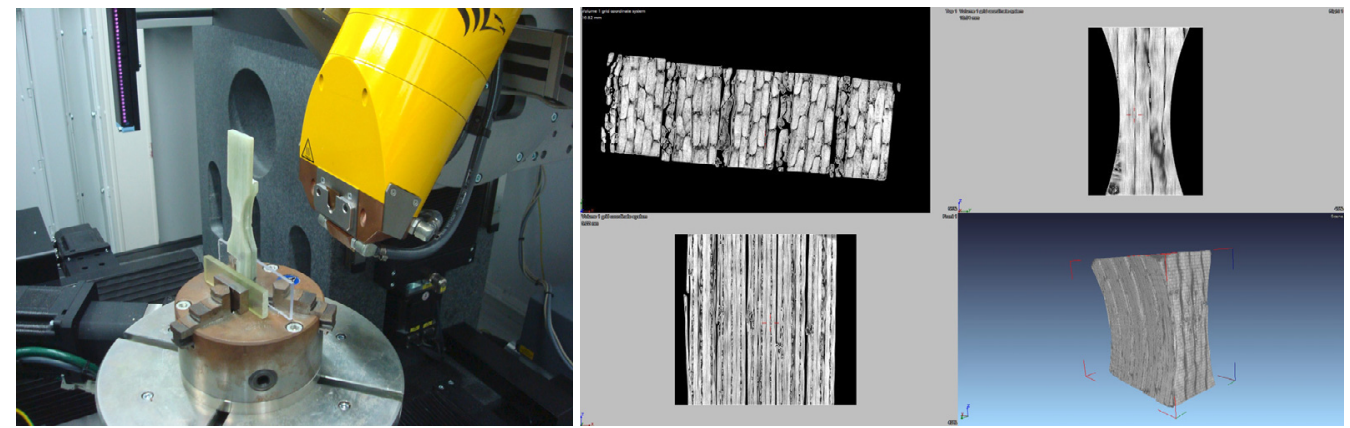

Fig. 4. (a) Montage d'analyse par micro-tomographie $X,(b)$ images du centre de 5 éprouvettes après différents nombres de cycles en fatigue.

Fig. 4. (a) X-ray micro-tomography anaylsis, (b) images of the centre of 5 specimens after different numbers of fatigue cycles.

de surface aux bords de l'éprouvette sans dégradation thermique locale.

L'analyse des modifications physicochimiques et des endommagements a été réalisée par des moyens classiques (microscopie optique électronique à balayage (MEB), analyse thermique (DSC), spectroscopie FTIR) et par micro-tomographie $X$, figure 4 a. Cette dernière a permis avant essai de vérifier la qualité des échantillons et ensuite de mettre en évidence les endommagements à l'intérieur des échantillons sans besoin de les découper, figure $4 \mathrm{~b}$.

\section{Résultats d'essais de fatigue}

Dans un premier temps des éprouvettes nonvieillies ont été testées en flexion quatre points à l'air et dans l'eau de mer. La figure 5 présente un exemple de résultat, sur des composites non-vieillis renforcés de verre $E$ et de verre $H P$. On note le décalage de la courbe $S-N$ pour les essais réalisés sur le verre $E$ en eau de mer par rapport aux mêmes essais à l'air. Ceci souligne la nécessité de réaliser la caractérisation dans des conditions proches de celles de l'application. Par contre pour le verre $H P$ les mêmes essais n'indiquent pas d'influence notable de l'environnement de l'essai sur les résultats.

\subsection{Influence du vieillissement sur le comportement statique}

L'immersion dans l'eau de mer entraîne une prise de masse qui dépend de la matrice et de l'interface fibre/matrice. Pour les matériaux étudiés ici c'est surtout la matrice qui contrôle la prise en eau et on peut caractériser les cinétiques par une loi de diffusion fickienne (Fig. 6).

Le type de fibre n'affecte pas la prise en eau de manière importante. Des vieillissements de plusieurs mois en eau de mer ont été réalisés à $60^{\circ} \mathrm{C}$, et on constate une réduction des propriétés après vieillissement, figure 7. Cette évolution dépend de la fibre, elle est notablement plus lente pour le verre $H P$.

Le mode de rupture observé en flexion évolue avec la durée de vieillissement. Initialement notée en compression, après une certaine durée d'immersion la rupture intervient en traction. Les évolutions des contraintes de rupture en traction et compression relevées lors d'essais uniaxiaux, figure $8 \mathrm{a}$, couplées avec un modèle fickien de diffusion d'eau, permettent de prédire ce changement [27], figure 8b. La modélisation a été réalisée avec le logiciel Comsol Multiphysics. Le comportement du matériau est orthotrope.

\subsection{Influence du vieillissement sur le comportement en fatigue}

Les résultats ci-dessus indiquent que les propriétés quasi-statiques de ces composites sont sensibles à l'eau de mer. Pour une pale d'hydrolienne qui est sollicité de manière cyclique il est important d'évaluer l'influence du vieillissement sur le comportement en fatigue. La figure 9 présente des courbes 


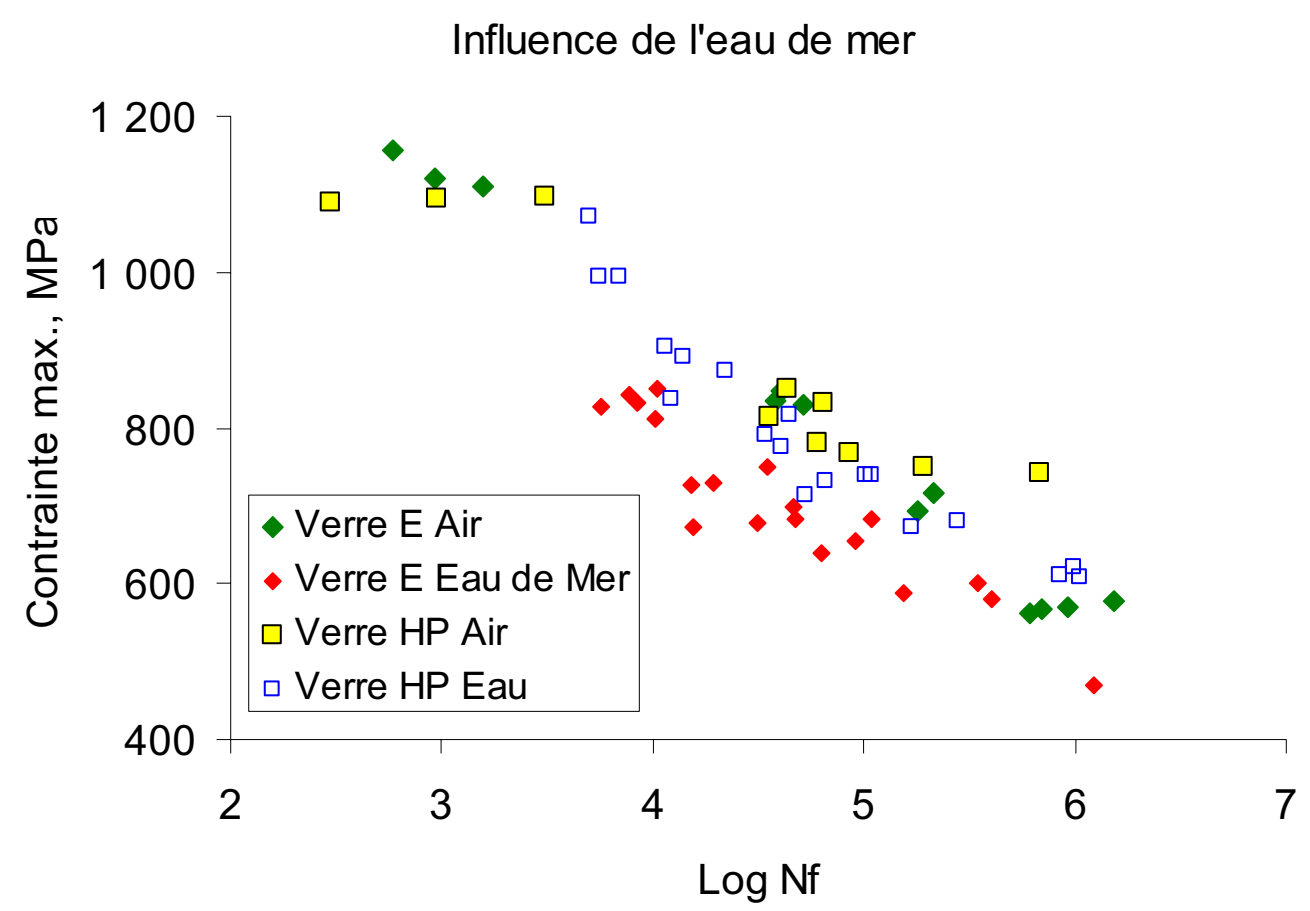

Fig. 5. Influence du milieu de l'essai sur le comportement en fatigue, Verre $E$ et $H P$, éprouvettes non-vieillies.

Fig. 5. Influence of test environment on fatigue behaviour, E-glass and HP glass, unaged specimens.

Prises d'eau, $60^{\circ} \mathrm{C}$

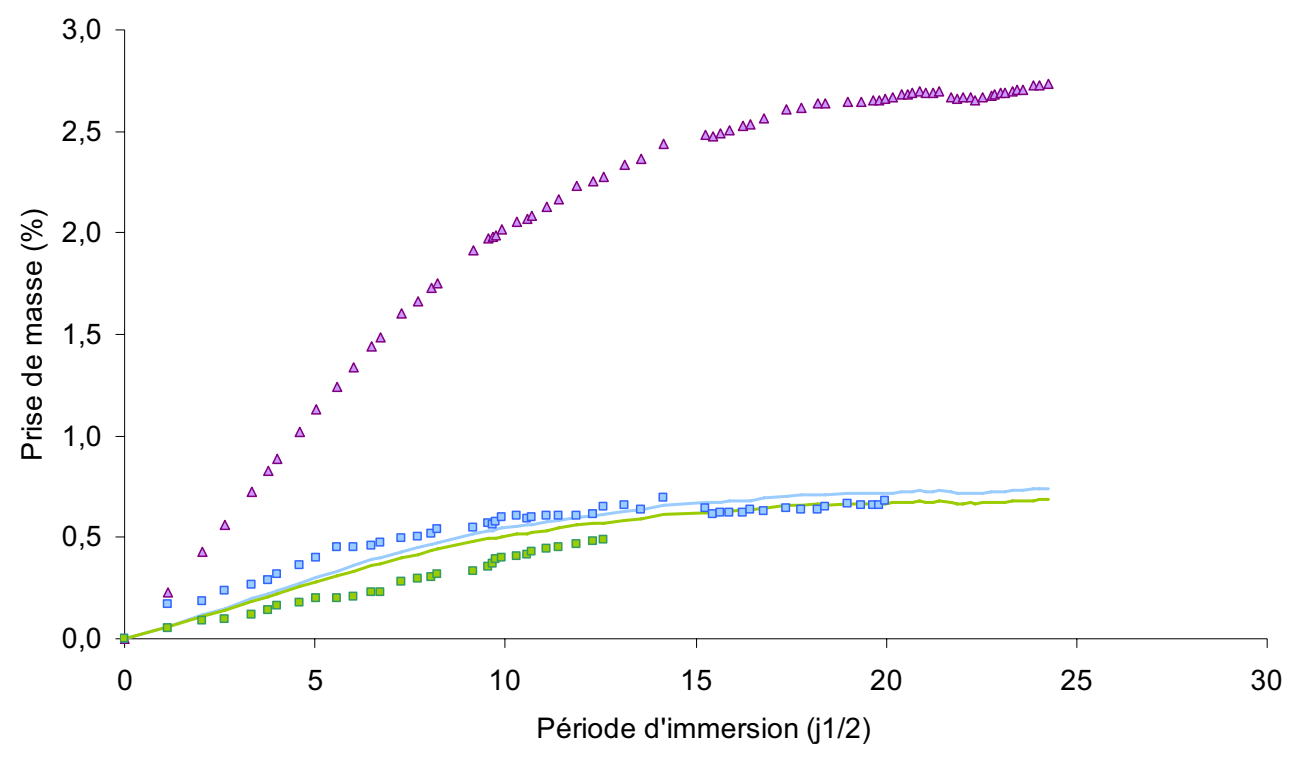

$\triangle$ Résine a Verre E [mesure] \Verre HP [mesure] — Verre E [calculé] — Verre HP [calculé]

Fig. 6. Prises d'eau, matrice et composite, $60^{\circ} \mathrm{C}$ eau de mer.

Fig. 6. Weight gains, matrix and composite, $60^{\circ} \mathrm{C}$ sea water. 


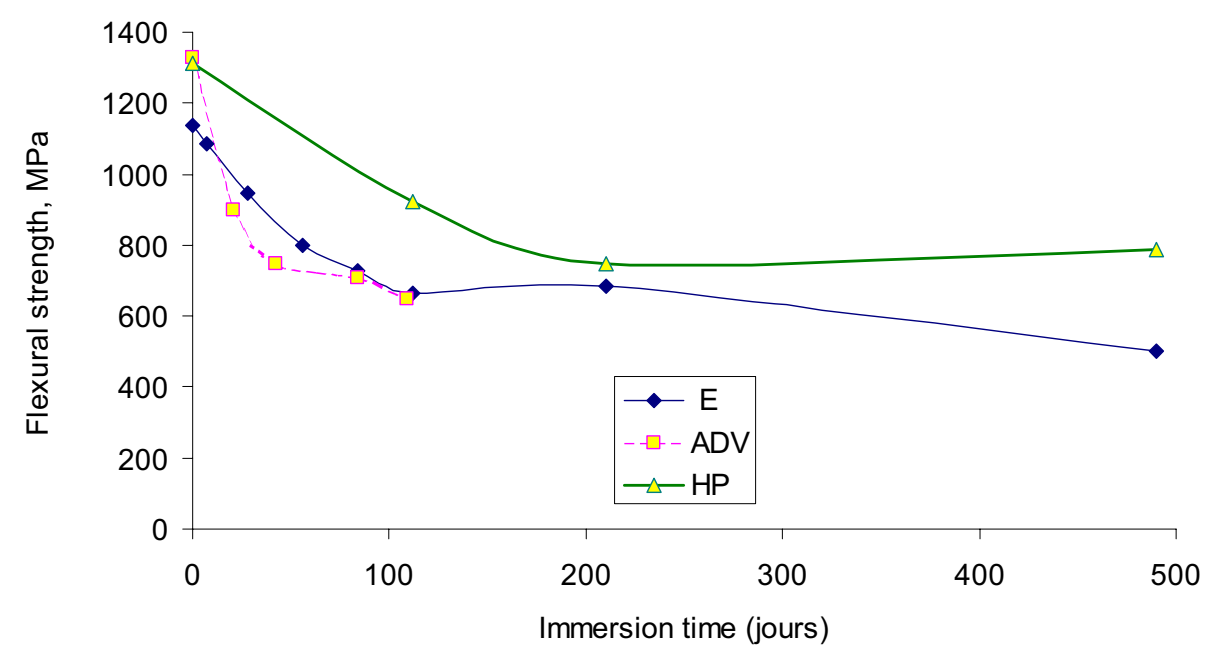

Fig. 7. Évolution de la résistance en flexion, 3 composites après vieillissement $60^{\circ} \mathrm{C}$ eau de mer. Fig. 7. Change in flexural strength of 3 composites after $60^{\circ} \mathrm{C}$ sea water ageing.

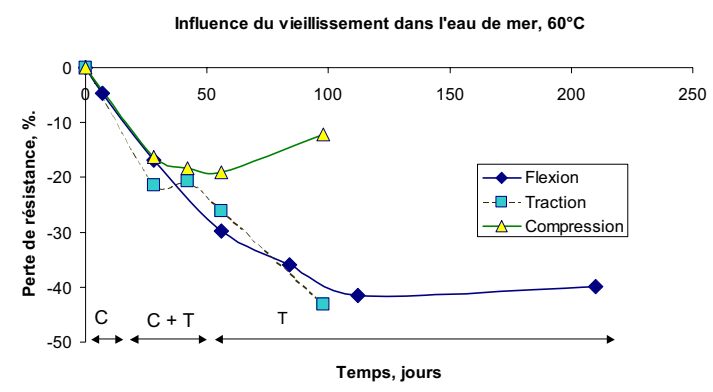

(a)

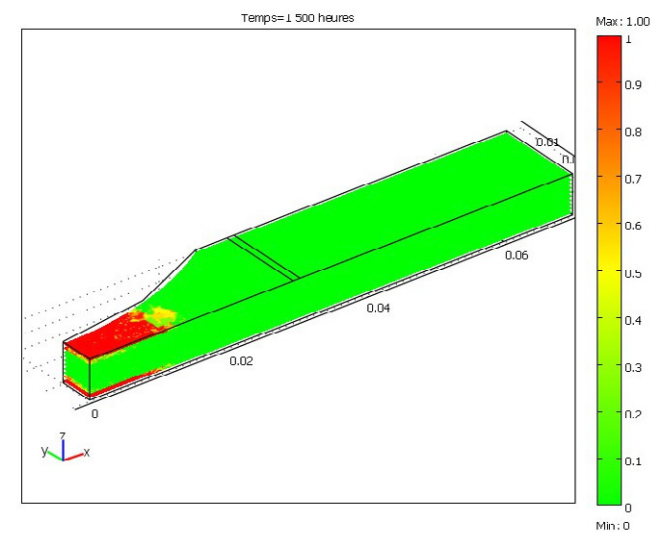

(b)

Fig. 8. (a) Évolution des résistances en flexion, traction et compression de composites verre $E$ après vieillissement, indiquant le changement de mode de rupture en flexion de Compression (C) à Traction (T), (b) Modèle numérique couplé diffusion/résistance mécanique.

Fig. 8. (a) Changes in flexural, tensile and compression strengths for E-glass after aging, showing failure mode (C: Compression, T: Tension), (b) numerical model with coupled diffusion and strength.

$S-\log N$ en eau de mer à $20^{\circ} \mathrm{C}$, d'un matériau préalablement vieilli 15 semaines en eau de mer à $60^{\circ} \mathrm{C}$, qui indiquent une forte influence du vieillissement sur la résistance en fatigue.

\subsection{Influence de la résine et de l'ensimage sur le comportement en fatigue}

Les résultats précédents (Fig. 5) indiquent que la durée de vie peut être prolongée par la sélection d'une fibre de verre aux proprié- tés améliorées par rapport à une fibre verre- $E$ standard, mais que cette amélioration reste faible par rapport à l'influence du vieillissement (Fig. 9). Il est donc intéressant d'examiner les rôles de la matrice et de l'interface fibre/matrice.

En ce qui concerne la matrice, la figure 10 présente un résultat pour les mêmes fibres ADV avec une résine alternative et un ensimage adapté à cette résine. Ici, on constate une plus faible variation du comportement en fatigue après les mêmes vieillissements, ce qui souligne la grande importance de la 
Influence du vieillissement, 15 semaines $60^{\circ} \mathrm{C}$ eau de mer

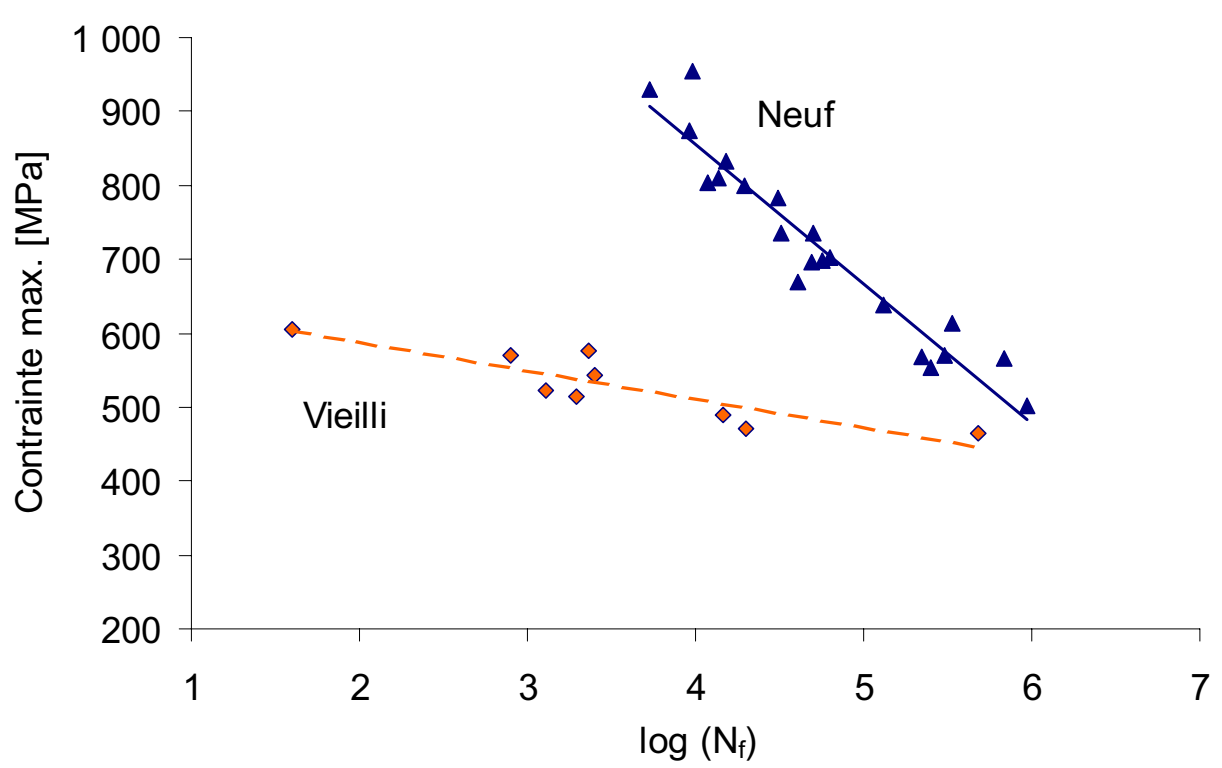

Fig. 9. Influence du vieillissement sur la résistance en fatigue, ADV/Epoxy.

Fig. 9. Influence of ageing on fatigue strength, ADV/Epoxy.

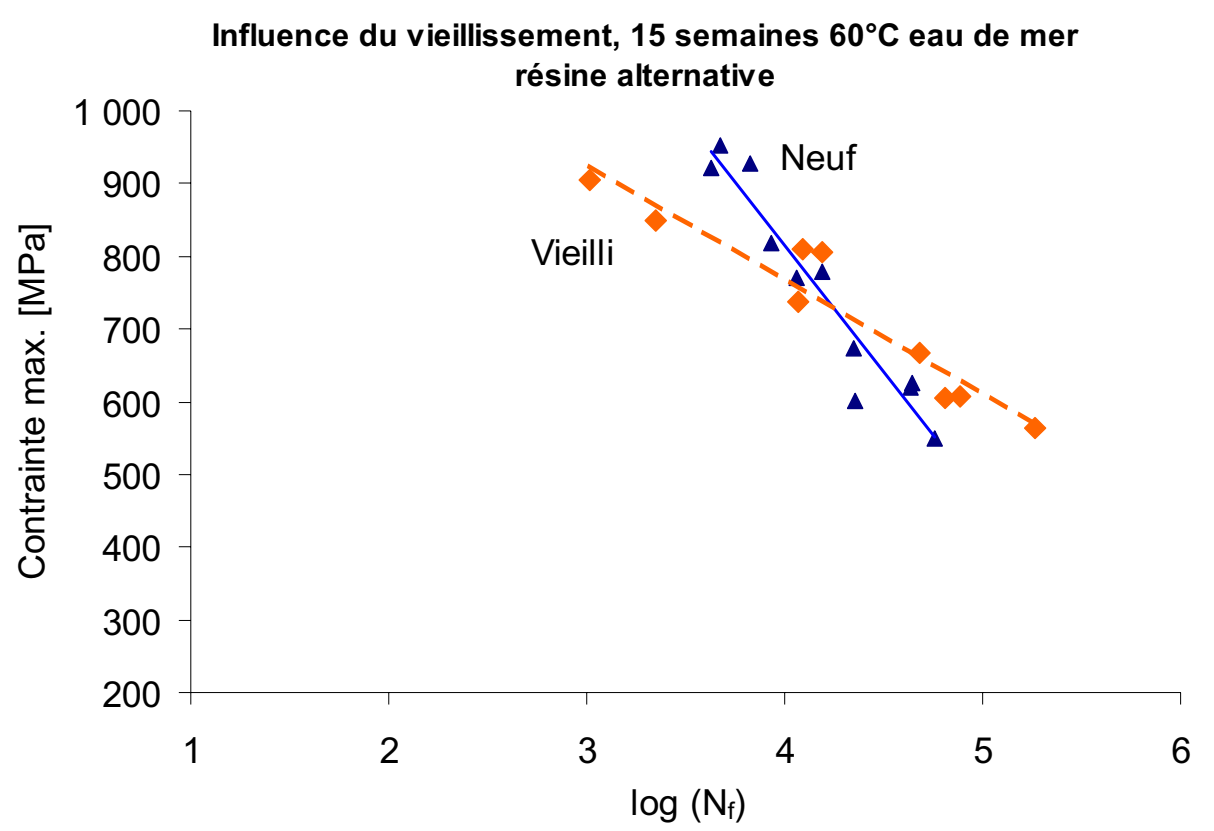

Fig. 10. Matrice alternative, mêmes fibres ADV, comportement en fatigue.

Fig. 10. Alternative matrix, same ADV fibres, fatigue behaviour.

formulation de la résine qui, bien que présentant des propriétés en fatigue à l'état non vieilli inférieures à la résine époxy semble présenter à l'état vieilli des propriétés supérieures.

Pour tous les essais décrits ci-dessus l'ensimage a été optimisé. La figure 11 présente quelques résultats pour un composite avec des fibres $H P$, soit avec un ensimage optimisé pour la matrice soit non-optimisé, et on constate que l'ensimage joue également un rôle important en fatigue. Ces essais ont été réalisés à un charge maximale cyclique de $60 \%$ de celle à la rupture.

Ces résultats confirment ceux présentés récemment par Watson [31], qui obtenait une 
a) Statique

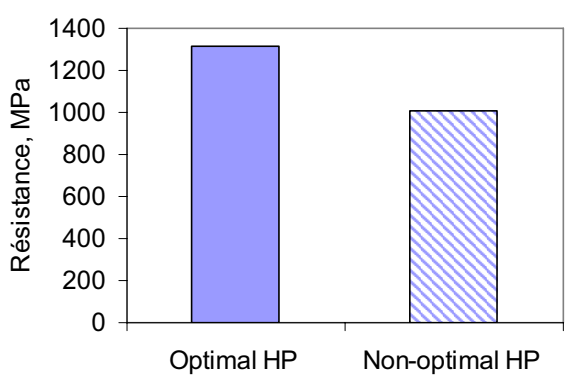

b) Fatigue

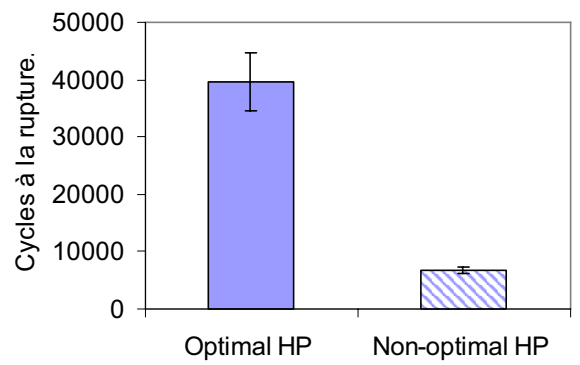

Fig. 11. Influence de l'ensimage, comportement en flexion, statique et fatigue.

Fig. 11. Influence of fibre sizing on static and fatigue behaviour in flexure.

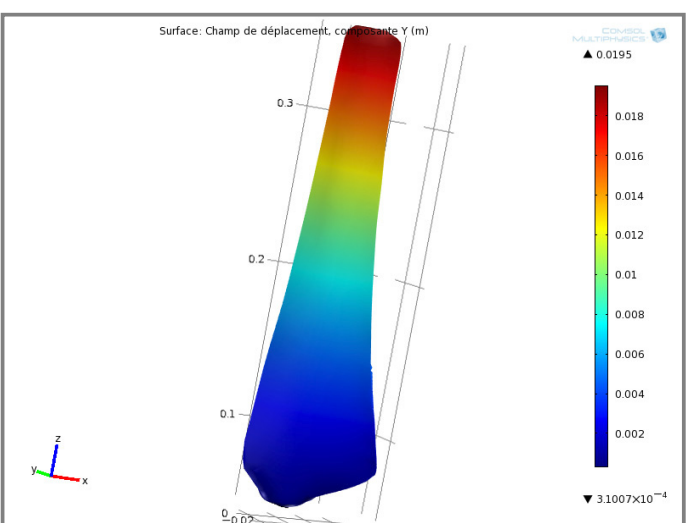

(a)

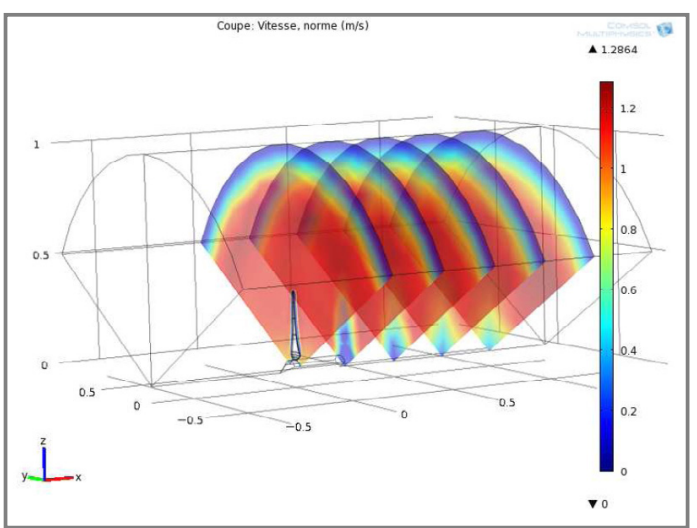

(b)

Fig. 12. (a) Modèle de pale, (b) modèle d'un tiers de la turbine soumis à un courant de vitesse $\mathbf{1}$ m/s. Fig. 12. (a) Numerical model of blade, (b) model of one third of turbine, current flow $1 \mathrm{~m} / \mathrm{s}$.

augmentation d'un facteur de 3 de la durée de vie en fatigue à l'air en optimisant la chimie de la surface de la fibre.

L'ensemble de ces résultats permet de mieux comprendre les paramètres qui gouvernent la durabilité des composites pour des structures EMR. Ceci est essentiel, non seulement pour mieux sélectionner les matériaux mais également pour optimiser la conception, afin de mieux définir les coefficients de sécurité. À cet effet, la modélisation par éléments finis de l'ensemble de la structure est abordée.

\section{Application à une hydrolienne}

À partir des résultats précédents, des modélisations numériques à différents niveaux (éprouvette, petite structure, couplage mécanique et diffusion) ont permis d'aboutir à la modélisation simplifiée d'une turbine complète à axe horizontal, figure 12 .

Ce type de modélisation permet d'évaluer l'influence des propriétés des matériaux et d'optimiser la géométrie du système avant de passer aux essais en mer. Une campagne d'essais en bassin hydrodynamique d'IFREMER à Boulogne sur une turbine avec des pales instrumentées, figure 13 , a permis de comparer les résultats d'essais avec les prédictions de la modélisation [32].

\section{Conclusions et Perspectives}

L'étude présentée brièvement ici a permis de dégager plusieurs conclusions :

- la performance de composites sollicités en flexion dans l'eau de mer dépend étroitement du type de fibre, de l'ensimage, et surtout de la formulation de la matrice ; 

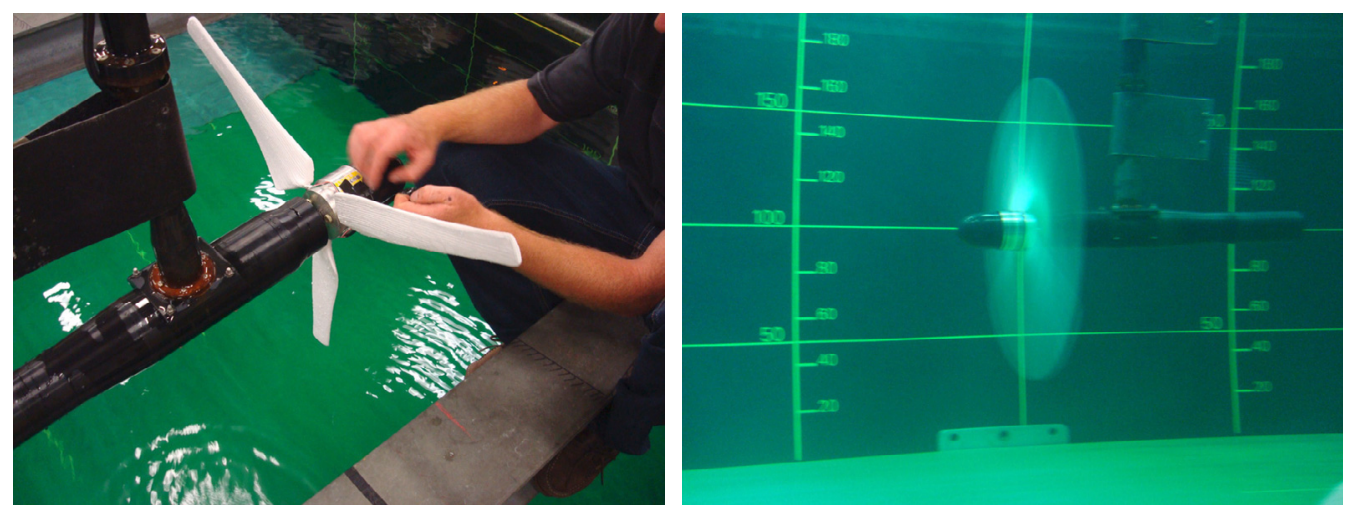

Fig. 13. Essais sur pales composites instrumentées au bassin.

Fig. 13. Tests on instrumented composite blades in wave tank.

- une procédure de vieillissement accéléré, liée à un protocole d'essai de fatigue dans l'eau de mer, permet maintenant de quantifier l'influence de ces composants et ainsi d'optimiser la réponse des composites sous chargement couplé de fatigue et d'immersion ;

- la mise en place de modèles numériques est une première étape dans l'intégration de la réponse du matériau dans la conception de structures de récupération d'énergie marine ;

- l'information qui manque à ce jour est une bonne connaissance des états de chargements en mer. L'étude de prototypes en bassin fournit une partie de cette information mais des essais en conditions réelles sont indispensables. Dans un avenir proche, l'instrumentation de pales en mer est prévue pour améliorer ces connaissances.

\section{Remerciements}

Les auteurs remercient Luc Peters de 3B, Claude Renaud et Paul Lucas d'OCV, et Rolf Nickel et Christoph Kensche de Momentive, pour leurs contributions importantes au projet. Les contributions de Nicolas Lacotte (essais de fatigue) et Grégory Germain (essais au bassin) d'IFREMER sont également très appréciées.

\section{Références}

[1] Ifremer, Synthèse de l'étude prospective sur les énergies renouvelables marines à l'horizon 2030, (March 2008), http://wwz.ifremer. fr/institut/Les-ressources-documentaires/ Publications/Documents-referents
[2] World Energy Council, Survey of Energy Resources, 2010

[3] Agence Internationale de l'énergie, IEA/OES, 2000

[4] R. Martin, editeur, Aging of Composites, Woodhead Publishing, 2008

[5] E. Greene, Marine composites Second Edition, Available from : http://www. marinecomposites.com/

[6] P. Davies, L. Lemoine, Nautical applications of composite materials, in 3rd IFREMER Conference, Paris, France, 1992

[7] G.S. Springer, ed. Environmental Effects on Composite Materials, Technomic Publishers, 1981

[8] P. Davies, R. Baizeau, D. Choqueuse, L. Salmon, F. Nagot, Aging and long term behavior of composite tubes, in Proceedings of Recent Developments in durability analysis of composite systems, Duracosys, 1999

[9] F. Richard, D. Perreux, Compos. Sci. Technol. 61 (2001) 2087-2094

[10] Y. Weitsman, Moisture in composites, in Reifsnider KL editor, Fatigue of composites, Elsevier, 1991, pp. 385-429

[11] E.P. Gellert, D.M. Turley, Compos. Part A 30 (1999) 1259-1265

[12] P. Davies, F. Mazeas, J. Compos. Mater. 35 (2001) 1343-1372

[13] G. Kotsikos, J.T. Evans, A.G. Gibson, J.M. Hale, Compos. Part A 31 (2000) 969-977

[14] B. Harris, ed. Fatigue in Composites, Woodhead Publishers, 2003

[15] J.F. Mandell, D.D. Samborsky, P. Agastra, Composite Materials Fatigue Issues in Wind Turbine Blade Construction, SAMPE 2008, Long Beach, 2008

[16] J.F. Mandell, D.D. Samborsky, P. Agastra, A.T. Sears, T.J. Wilson, Analysis of SNL/MSU/DOE Fatigue Database Trends for Wind Turbine Blade Materials, Contractor Report SAND2010-7052, Sandia National Laboratories, Albuquerque, NM, 2010 
[17] A.T. Echtermeyer, C. Kensche, P. Bach, M. Poppen, H. Lilholt, S. Andersen, P. Bronsted, Method to predict fatigue lifetimes of GRP wind turbine blades and comparison with experiments. in Proceedings of the 1996 European Union wind energy conference, Göteborg, Sweden, 1996

[18] R.P.L. Nijssen, A.M. VanWingerde, D.R.V. VanDelft, S.A.M.P.E. Journal 43 (2007) 7-15

[19] OPTIMAT, base de données, http://www. wmc.eu/optimatblades_optidat.php

[20] G. Gardiner, Tidal Turbines to Mine Marine Megawatts, Composite Technology, 2007

[21] P.L. Fraenkel, Development and testing of Marine Current Turbine's SeaGen1.2MW tidal stream turbine, Proc. 3rd International Conference on Ocean Energy, October 2010, Bilbao

[22] Open Hydro Press release, December 2010, http://www.openhydro.com/news/171210. html

[23] P. Bornens, J.F. Daviau, L. Gaillard, A. Guerrier, J. Ruer, The Sabella Tidal Turbine - Tests Results and Further Development, Proc. 3rd International Conference on Ocean Energy, October 2010, Bilbao

[24] S. Antheaume, T. Maître, J.-L. Achard, Renewable Energy 33 (2008) 2186-2198

[25] J. Zanette, D. Imbault, A. Tourabi, Renewable Energy 35 (2010) 997-1009
[26] G. Marsh, Reinforced Plastics 48 (2004) 44-47

[27] Y.J. Weitsman, D. Penumadu, A. Sirriuk, On the immersed and dry fatigue of carbon fiber/vinyl ester composite material, Proc ICCM17, 2009

[28] V. Pauchard, Étude des mécanismes de rupture des fibres dans les composites verre/epoxy sous sollicitation de fatigue longitudinale en milieu humide Thèse de doctorat, École Centrale de Lyon, 2001

[29] A. Boisseau, Long term durability of composites for ocean energy conversion systems, Thèse de doctorat, Université de Franche Comté, 2011

[30] A. Boisseau, P. Davies, Choqueuse et al., Seawater Aging of Composites for Ocean Energy Conversion Systems Proc. ICCM17, Edinburgh, 2009

[31] J.C. Watson, SAMPE Journal 46 (2010) 8-15

[32] P. Davies, G. Germain, B. Gaurier, A. Boisseau, D. Perreux, Evaluation of the durability of composite tidal turbine blades, Proc. Ninth European Wave and Tidal Energy Conference (EWTEC2011), University of Southampton, UK, 2011 